\title{
The concurrent stimulation of Wnt and FGF8 signaling induce differentiation of dental mesenchymal cells into odontoblast-like cells
}

\author{
Motoyoshi Kimura $^{1} \cdot$ Akiko Saito $^{2} \cdot$ Shoko Onodera $^{2} \cdot$ Takashi Nakamura $^{2,3} \cdot$ Makoto Suematsu $^{3} \cdot$ Seikou Shintani $^{1}$. \\ Toshifumi Azuma² ${ }^{\circledR}$
}

Received: 16 April 2021 / Accepted: 13 June 2021 / Published online: 5 November 2021

(c) The Author(s) 2021

\begin{abstract}
Fibroblast growth factor 8 (FGF8) is known to be a potent stimulator of canonical Wnt//-catenin activity, an essential factor for tooth development. In this study, we analyzed the effects of co-administration of FGF8 and a CHIR99021 (GSK3 $\beta$ inhibitor) on differentiation of dental mesenchymal cells into odontoblasts. Utilizing Cre-mediated EGFP reporter mice, dentin matrix protein 1 (Dmp1) expression was examined in mouse neonatal molar tooth germs. At birth, expression of Dmp1-EGFP was not found in mesenchymal cells but rather epithelial cells, after which Dmp1-positive cells gradually emerged in the mesenchymal area along with disappearance in the epithelial area. Primary cultured mesenchymal cells from neonatal tooth germ specimens showed loss of Dmp1-EGFP positive signals, whereas addition of Wnt3a or the CHIR99021 significantly regained Dmp1 positivity within approximately 2 weeks. Other odontoblast markers such as dentin sialophosphoprotein (Dspp) could not be clearly detected. Concurrent stimulation of primary cultured mesenchymal cells with the CHIR99021 and FGF8 resulted in significant upregulation of odonto/osteoblast proteins. Furthermore, increased expression levels of runt-related transcription factor 2 (Runx2), osterix, and osteocalcin were also observed. The present findings indicate that coordinated action of canonical Wnt/ $\beta$-catenin and FGF8 signals is essential for odontoblast differentiation of tooth germs in mice.
\end{abstract}

Keywords Dental mesenchymal cells · Canonical Wnt · FGF8 · Odontoblast · Tooth development

\section{Introduction}

Tooth development is initiated by thickening of oral epithelium and adjacent cranial neural crest (CNC) derivedmesenchyme, after which this epithelial-mesenchymal complex becomes invaginated into the underlying $\mathrm{CNC}$ to form a tooth bud. The mesenchyme then starts to express a specific set of transcription factors and signaling molecules, and gains an ability to induce tooth morphogenesis.

Toshifumi Azuma

tazuma@tdc.ac.jp

1 Department of Pediatric Dentistry, Tokyo Dental College, 2-9-18 Kanda-Misaki-Chou, Chiyoda, Tokyo 101-0061, Japan

2 Department of Biochemistry, Tokyo Dental College, 2-9-18 Kanda-Misaki-Chou, Chiyoda, Tokyo 101-0061, Japan

3 Department of Biochemistry, Keio University School of Medicine, 35 Shinanomachi, Shinjukuku, Tokyo 160-8582, Japan
Eventually, the epithelium differentiates into ameloblasts and mesenchyme into odontoblasts [1,2]. The molecular regulation of early tooth morphogenesis leading to tooth bud formation has been extensively studied. Indeed, a large body of work shows that the network controlling tooth development includes major signaling pathways, such as those of transforming growth factor $\beta$ (TGF $\beta$ ), BMP, FGF, Wnt, and $\mathrm{SHH}$, indicating recurrent functions at various stages $[3,4]$. However, the precise molecular network controlling the late stages of tooth development including odontoblast differentiation is very complex and yet to be determined [5], and there is a need to provide more comprehensive understanding of odontoblast development.

Odontoblasts do not repair dentin in vivo despite their ability to create new dentin throughout life in response to damage by stem cells, termed dental pulp stem cells (DPSCs), which show similarities to human bone marrow stromal cells (BMSCs). DPSCs are capable of migrating to the dentin surface and differentiating into odontoblasts to form reparative dentin. However, unlike primary dentin, 
this reparative dentin is poorly organized, with irregular dentinal tubules embedded in the dentin matrix. It is also not yet known how primary dentin is organized by CNCderived mesenchymal cells, while the genetic basis for odontogenic ability remains to be explained for dental epithelium and mesenchyme. The currently understood gene expression signature of dental mesenchyme during the early morphogenetic stage includes numerous potential candidates that might account for odontogenic competence of the tissue [6]. In particular, de novo tooth formation has been induced in transgenic mice by stimulating the canonical Wnt pathway, with constitutive expression of $\beta$-catenin in the ectoderm resulting in formation of extra teeth [7]. Therefore Wnt signaling, particularly canonical Wnt pathways, might have odontogenic inducing potential [8-10].

Several members of the FGF family are expressed during early development of the tooth germ and function at a definite stage of tooth development from the start to finish of tooth formation. Intensive FGF8 expression is initially detected in dental epithelium, presumably before onset of tooth formation, and then persists there until the early bud stage. FGF signaling is involved in limiting the site of tooth formation by inducing expressions of Paired box gene 9 (Pax9), Paired-like homoedomain 1 (PitxI), and Paired-like homoedomain 2 (Pitx2) [11]. FGF8 is involved in induction of BarH-like homeobox 1 (Barxl) in the expected intermolecular space [12], and also responds primarily to LIM/homeobox protein 6 (Lhx6) and LIM/ homeobox protein $7(L h x 7)$ expression in odontogenic mesenchyme before initiation of and during tooth formation [13]. However, it remains unclear whether FGF8 is a component of the suggestive odontogenic potential in oral epithelium, as in mice lacking FGF8 in the oral epithelium, most of the architecture including molars is used to form tooth germs, but not teeth. Another FGF, presumably FGF9, may rescue incisor formation in the absence of FGF8 [12]. It has also been speculated that FGF8 is involved in induction of FGF3 expression in tooth mesenchyme [14]. Therefore, though FGF8 is important for tooth development, its detailed mechanism is largely unknown. Presently, differentiation and maintenance of odontoblast cultures are difficult to perform, thus the effects of Wnt and FGF8 on odontoblast differentiation and maintenance remain to be elucidated.

In the present study, Dmp1-Cre-recombinase transgenic mice, developed by crossing with CAG-CAT-EGFP mice, were utilized as a reporter strain to detect Dmp1 expression in dental germ mesenchymal cells. In newly born mice, dental germ epithelial cells expressed Dmp1, while mesenchymal cells did not, though at 4 days after birth, dental germ mesenchymal cells began to express Dmp1. We also found that canonical Wnt signaling plays a key role in Dmpl expression in dental germ-derived mesenchymal cells. These findings suggest that the role of the Wnt canonical pathway is important in late stage odontogenesis.

Addition of FGF8 enhanced the degree of differentiation of tooth germ-derived mesenchymal cells into odontoblasts. Furthermore, some bone-related markers were increased, whereas others were decreased. An increase or decrease in Runx 2 may have effects on the border of differentiation into osteoblast/cyte or odontoblast. The present results show that interaction between the Wnt canonical pathway and FGF8 is important for odontoblast differentiation, providing new evidence for elucidation of the mechanism of tooth development.

\section{Materials and methods}

\section{Animals}

Dmpl-Cre mice were crossed with a transgenic mouse line carrying a reporter gene construct known as CAG-CATEGFP reporter mice [15] to obtain Dmpl-EGFP mice. All mice were anesthetized using isoflurane (Abbvie, North Chicago) before decapitation for tooth germ harvesting.

\section{Tissue preparation}

Mandibles and first molars were taken from Dmp1-EGFP neonatal mice and fixed overnight in $4 \%$ paraformaldehyde phosphate buffer solution (Wako Pure Chemical Industries, Ltd., Osaka). Fixed tissues were then decalcified with $10 \%$ ethylenediaminetetraacetic acid (EDTA) (MUTO PURE CHEMICALS Co., Ltd., Tokyo) for 1 week. After decalcification, they were dehydrated with a series $(10 \%, 20 \%, 30 \%)$ of sucrose (Sigma-Aldrich Co. LLC, St. Louis) and phosphate buffered saline (PBS) (Takara Bio Inc., Shiga). Tissues were embedded in optimal cutting temperature compound (O.C.T., Sakura Finetek Japan Co., Ltd., Tokyo) and 10- $\mu \mathrm{m}$ sections were obtained using a cryostat (Leica, Wetzlar). Fluorescent imaging was performed using a fluorescence microscopy system (BZ-X700) (KEYENCE Corp., Osaka).

\section{Cell cultures}

Newborn mouse dental mesenchymal tissues were isolated from dental epithelium, then digested in $10 \%$ collagenase I (F. Hoffmann-La Roche, Ltd., Basel) for $2 \mathrm{~h}$ and $0.05 \%$ trypsin-EDTA (Thermo Fisher Scientific Inc., Waltham) for $8 \mathrm{~min}$ at $37^{\circ} \mathrm{C}$. Enzymatically digested tissues were washed three times with Dulbecco's modified Eagle's medium (DMEM) (Thermo Fisher Scientific Inc., Waltham) containing 10\% fetal bovine serum (FBS) (Biosera Inc., Kansas City) and $1 \%$ penicillin streptomycin (Thermo Fisher Scientific Inc., Waltham). Murine dental mesenchymal cells were 
cultured in DMEM supplemented with 10\% FBS and 1\% penicillin streptomycin at $37{ }^{\circ} \mathrm{C}$ in a humidified atmosphere containing $5 \% \mathrm{CO}_{2}$.

Cells were cultured in 12-well plates at a density of $1.0 \times 10^{4}$ cells per well, then $50 \mathrm{ng} / \mathrm{ml} \mathrm{Wnt3a}$ (R\&D Systems, Inc., Minneapolis), $50 \mathrm{ng} / \mathrm{ml} \mathrm{Wnt5a} \mathrm{(R \& D} \mathrm{Systems,}$ Inc., Minneapolis), 25 ng/ml FGF8 (R\&D Systems, Inc., Minneapolis), and $3 \mu$ M CHIR99021 (Sigma-Aldrich Co. LLC, St. Louis) were added, and culturing was continued for 3 weeks to induce differentiation. The concentration of each chemical regent was determined based on previous papers [16-19]. The medium, which contained cytokines, was changed every 2 days.

\section{Immunohistochemistry}

Cells were fixed with $4 \%$ paraformaldehyde (Wako Pure Chemical Industries Ltd., Osaka) for $20 \mathrm{~min}$ at room temperature and rinsed three times with PBS. Fixed cells were permeabilized with $0.1 \%$ Triton X-100 (Wako Pure Chemical Industries, Ltd., Osaka) in PBS for 10 min. After membrane permeabilization, the cells were blocked with 5\% goat serum (Wako Pure Chemical Industries, Ltd., Osaka) in PBS for $15 \mathrm{~min}$, then incubated with primary antibodies, including monoclonal chicken antibody conjugated with anti-GFP IgG (Cell Signaling Technology, Inc., Danvers) and monoclonal rabbit antibody conjugated with anti- $\beta$-catenin IgG (Cell Signaling Technology, Inc., Danvers) at a dilution of 1:100 for $1 \mathrm{~h}$ at room temperature. After washing with cold 5\% goat serum in PBS three times, the cells were treated with secondary goat anti-chicken Alexa Fluor 488 (Cell Signaling Technology, Inc., Danvers) or goat anti-rabbit Alexa Fluor 594 (Cell Signaling Technology, Inc., Danvers) at a dilution of 1:1000 for $1 \mathrm{~h}$ at room temperature. Fluorescent imaging was performed using a fluorescence microscopy system (BZX700) (KEYENCE Corp. Osaka).

\section{RNA isolation and quantitative PCR}

Total RNA was isolated from each sample using QIAzol Lysis Reagent (Qiagen, Inc., Hilden) and RNA purity was assessed with a NanoDrop ${ }^{\circledR}$ ND-1000 spectrophotometer (Thermo Fisher Scientific, Waltham, MA). cDNA was synthesized using a cDNA Reverse Transcription Kit (Thermo Fisher Scientific Inc., Waltham), according to the manufacturer's instructions. qRT-PCR analysis was performed using Premix Ex Taq ${ }^{\mathrm{TM}}$ reagent (Takara Bio Inc., Otsu), according to the manufacturer's protocol, and an Applied Biosystems ${ }^{\circledR}$ 7500 Fast Real-Time PCR System. The primer sequences are listed in Table 1. Reactions were performed for 40 cycles of $5 \mathrm{~s}$ each at $95{ }^{\circ} \mathrm{C}$ and 34 cycles at $60 \mathrm{~s}$. Relative mRNA expression was determined using the $\Delta \Delta \mathrm{CT}$ method.

\section{Flow cytometry analysis}

Dental mesenchymal cells were harvested after culturing for 3 weeks using $0.05 \%$ trypsin and suspended in PBS containing 10\% FBS. EGFP-positive cells were isolated using a FACSAria system (Becton, Dickinson and Company, Franklin Lakes).

\section{Statistical analysis}

Quantitative values are expressed as the mean \pm SD. Oneway ANOVA was performed using the SPSS-Windows software package, v. 14.0 (SPSS Inc., Chicago). $P$ values less than 0.01 were considered to indicate statistical significance.
Table 1 Primers used for quantitative reverse transcription polymerase chain reaction (qRT-PCR)

\begin{tabular}{llll}
\hline Gene symbol & Left & Right & GenBank accession no \\
\hline$\beta$-actin & ccaaccgtgaaaagatgacc & accagaggcatacagggaca & NM_007393.5 \\
Alp & aatgaggtcacatccatcctg & cacccgagtggtagtcacaa & NM_007431.2 \\
$\beta$-catenin & tgcagatcttggactggaca & aagaacggtagctgggatca & NM_001165902.1 \\
Bglap & tgaggaccatctttctgctca & tggacatgaaggctttgtca & NM_007541.3 \\
Bsp & gaaaatggagacggcgatag & cattgtttcctcttcgtttga & NM_008318.2 \\
Dmp1 & cgctgaggtttgaccttgt & ttgggatgcgattcctctac & NM_016779.2 \\
Dspp & agccagtcagaagcatgtcc & ccttgttgggaccttcagt & NM_010080.3 \\
Nestin & tcccttagtctggaagtggcta & ggtgtctgcaagcgagagtt & NM_016701.3 \\
Runx2 & cgtgtcagcaaagcttcttt & ggctcacgtcgctcatct & NM_001145920.2 \\
Panx3 & gaaatctctctggcctcacaa & atacatggccacagccaga & NM_172454.2 \\
Sp7 & ctcctgcaggcagtcctc & gggaagggtgggtagtcatt & NM_130458.3 \\
\hline
\end{tabular}




\section{Results}

\section{Dmp1-EGFP expression in conditional transgenic murine odontoblasts and ameloblasts}

There was nearly no EGFP expression detected in the dental mesenchyme area in newborn Dmp1-EGFP mice, while that was noted in ameloblast areas (Fig. 1a-c). Two days after birth, Dmp1-EGFP signals were observed in the layer where odontoblasts were located (Fig. 1d, e). Furthermore, we also observed that EGFP signals in alveolar bones became stronger, whereas Dmp1-EGFP signals gradually became weaker in ameloblasts (Fig. 1g-O).

\section{Activation of canonical Wnt signals and increased Dmp1 expression}

Wnt signals are thought to function as essential developmental modulators of ecto-mesodermal development. We investigated canonical and non-canonical Wnt signaling to determine the effects on odontoblastic differentiation. Primary cultured tooth germ mesenchymal cells were obtained from newborn Dmp1-EGFP mice. We observed no Dmp1-EGFP signals in tooth mesenchymal cells in newly born mouse tooth germ mesenchymal cells. Morphological alterations were visible following administration of Wnt3a and especially the following that of CHIR99021, while cells cultured with the latter demonstrated a fibroblast-like spindle shape similar to that of odontoblasts. Administration of Wnt5a to activate the non-canonical Wnt pathway caused no obvious changes as compared to the control (Fig. 2a). Cells treated with CHIR99021 showed greater cell proliferation as compared with Wnt3a- or Wnt5a-treated cells (Fig. 2b). Following treatment with Wnt3a, CHIR99021, and Wnt5a, Dmpl expression was remarkably induced by CHIR99021. Also, Dspp expression was substantial in day 0 cultured cells, but was remarkably reduced to an undetectable level soon after beginning culturing. On the other hand, administrations of CHIR99021 and Wnt3a were able to maintain Dspp expression to some extent in cultured tooth germ mesenchymal cells (Fig. 2c). Immunofluorescence microscopy showed that administrations of CHIR 99021 and Wnt3a induced Dmp1-EGFP expression, especially CHIR99021 treatment, whereas Wnt5a failed to induce that. Nuclear $\beta$-catenin localization indicating Dmp1EGFP expression was also found to be induced by canonical Wnt signaling (Fig. 3).
Differentiation into odontoblasts by CHIR99021 and FGF8 concurrent treatment

While Dmpl expression was induced by CHIR99021 treatment of tooth germ mesenchymal cells, Dspp expression was sharply decreased after primary culturing of tooth germ mesenchymal cells, thus we concurrently treated the cells with CHIR99021 and FGF8. Phase imaging showed that FGF8 caused cell growth and the cell morphology had a cobblestone-like appearance, while CHIR99021 treatment induced a spindle-like morphology. Interestingly, simultaneous treatment with both FGF8 and CHIR99021 resulted in a spindle-like shape similar to that of odontoblasts (Fig. 4a). In addition, cells with both FGF8 and CHIR99021 added showed greater cell proliferation as compared to those with a single administration (Fig. 4b). Furthermore, flow cytometric analysis showed that tooth mesenchymal cells treated with both CHIR99021 and FGF8 induced differentiation to Dmp1-EGFP-positive cells (Fig. 5a), and the number of Dmp1-EGFP-positive cells were significant upregulated following treatment with both as compared to the non-treated group. (Fig. 5b).

qRT-PCR analyses revealed remarkable levels of expression of Dmp1, Dspp, Nestin, Pannexin3, Bsp, and Osterix (Osx) following treatment with CHIR99021 and FGF8 as compared with either administered alone. Also, treatment with CHIR99021 alone caused an increase in expression of Pannexin3 and Bone sialoprotein (Bsp), indicating promotion of canonical Wnt pathway activation. On the other hand, no increase in expression of any of these factors was seen with FGF8 alone (Fig. 6a, b). Together, these results indicate that canonical Wnt activation along with FGF8 significantly induces odontogenesis of tooth germ mesenchymal cells.

\section{Discussion}

In the present study, three main results were obtained. First, neonatal molar mesenchymal cells lost the odontoblastic phenotype immediately after cultivation. Second, canonical Wnt/ $\beta$-catenin signaling had some positive effects on odontoblast differentiation. Third, simultaneous treatment with canonical CHIR99021 and FGF8 gave rise to promotion of odontoblastic differentiation.

Although several reports have been presented indicating that mesenchymal odontoblastic differentiation relies nearly entirely on signals from adjacent epithelial cells [4, 9, 20-22], little is known about the molecular events that cause mesenchymal stem cells to proliferate and differentiate into odontoblast-like cells [23]. Thus, the main points of investigation in this study were determination of signals that induce mesenchymal odontoblast genesis and how those signals function. 


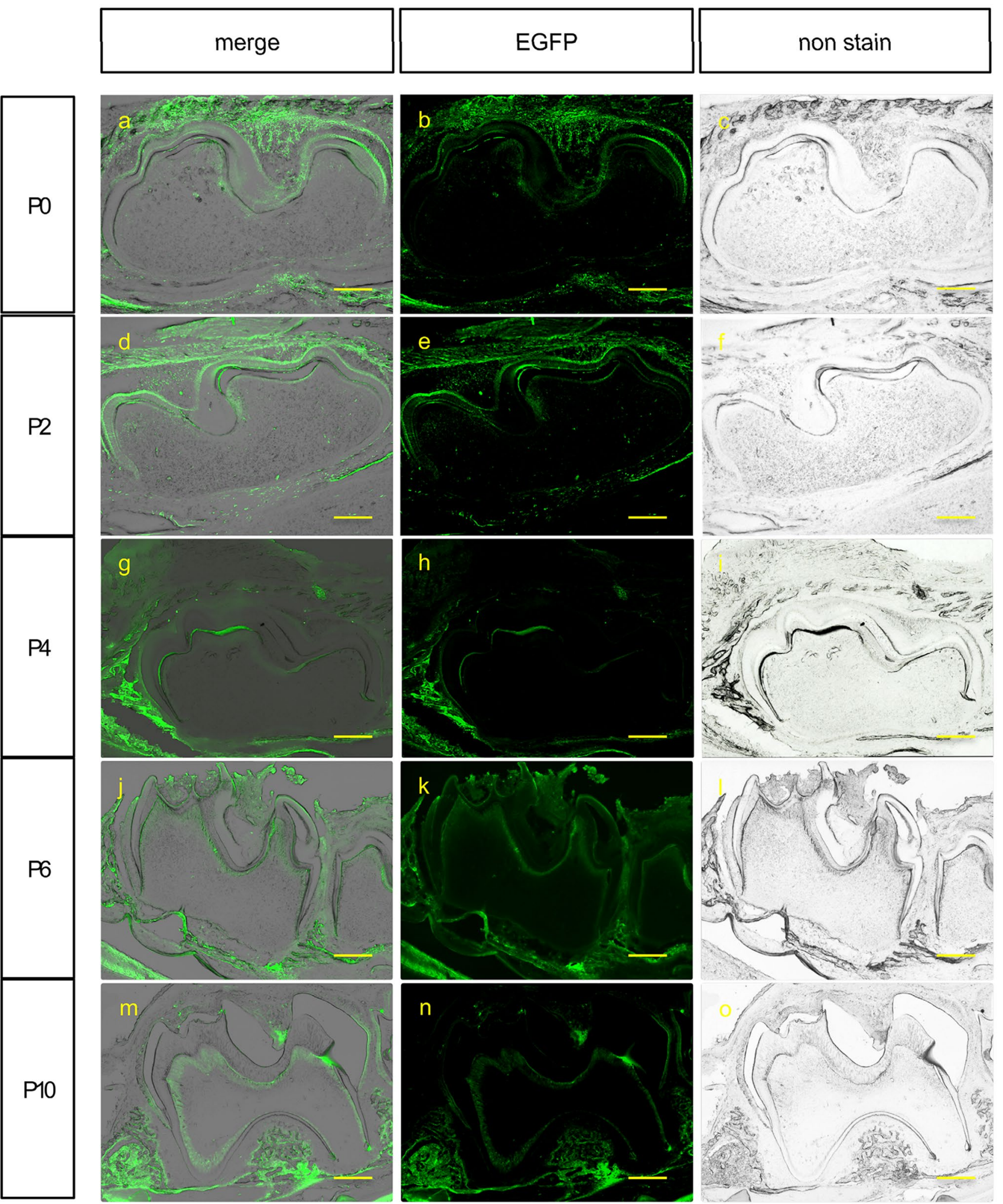

Fig. 1 Evaluation of Dmp1-EGFP expression in conditional transgenic mice tooth germs. Photographs show M1 of mice immediately following birth and on days 2, 4, 6, and 10. Dmp1-EGFP expression was observed in postnatal tooth epithelium, which shifted to the mes- enchyme with growth. The range of Dmp1-EGFP expression gradually expanded in the mesenchyme, while expression intensity in the tooth epithelium gradually decreased. Scale bar $=200 \mu \mathrm{m}$ 
Fig. 2 Activation of canonical Wnt signaling pathway increases osteogenic potential. a Morphology of tooth mesenchymal cells cultured with or without Wnt3a, Wnt5a, or CHIR99021. Morphological alterations were visible following administration of Wnt3a and especially CHIR99021. Cells cultured with CHIR99021 demonstrated a fibroblastlike spindle shape similar to that of odontoblast. Scale bar $=200 \mu \mathrm{m}$. b Growth curves of tooth mesenchymal cells with or without Wnt3a, Wnt5a, or CHIR99021. Values are shown as the mean \pm SD from three independent experiments. $* p<0.01$. c Dmp1 expression was markedly increased following administration of the CHIR99021. On the other hand, Dspp expression was prominently decreased in all of the samples. $* p<0.01$ a

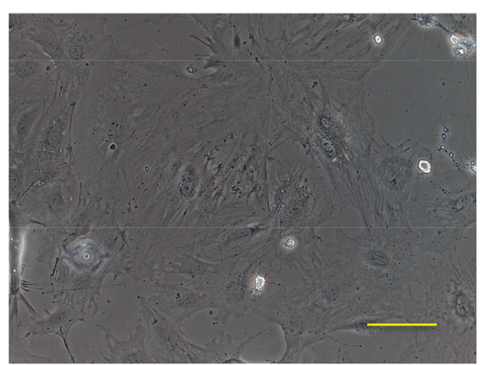

Control

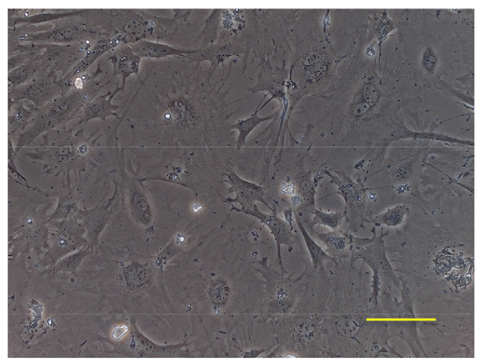

Wnt3a

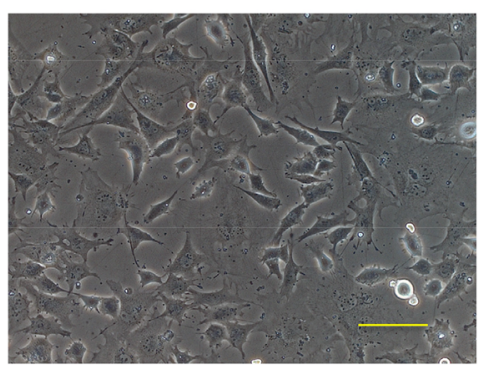

CHIR99021

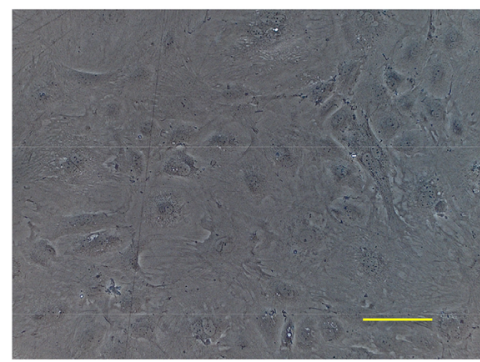

Wnt5a b

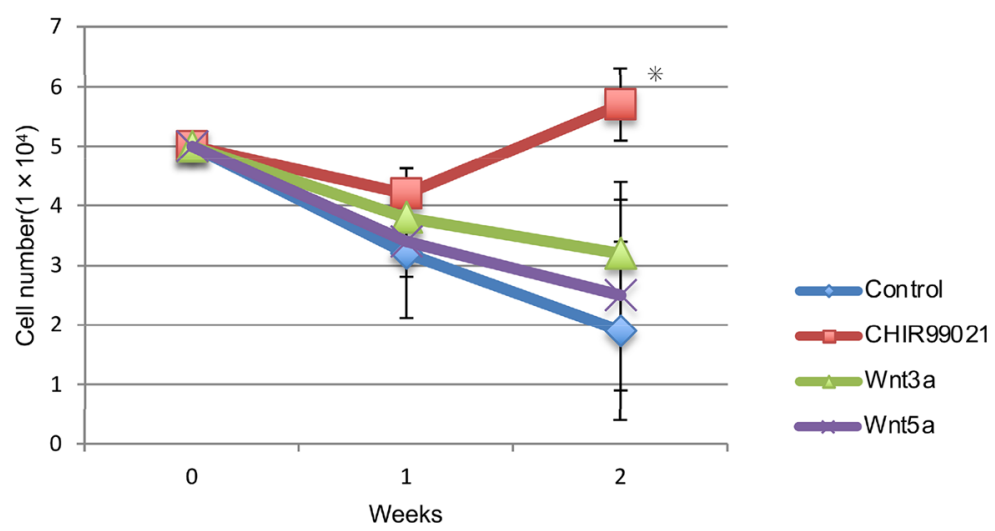

C

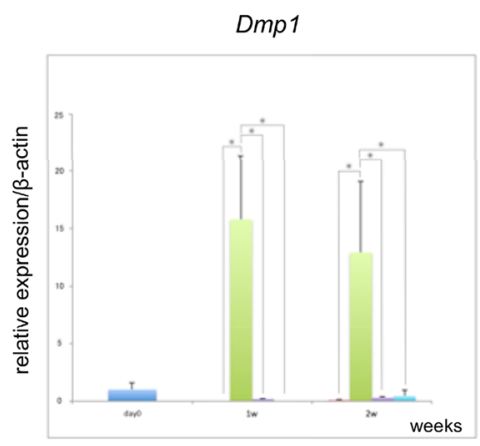

= day0

- Control - CHIR99021 -Wnt3a Wnt5a
The canonical Wnt/ $\beta$-catenin signaling pathway is important for stem cell renewal, proliferation, and differentiation $[24,25]$. During tooth development, Wnt/ $\beta$-catenin signaling is required at various stages of tooth morphogenesis. In the canonical Wnt / $\beta$ catenin signaling pathway, gene expression is regulated by expression of $\beta$-catenin in the cytoplasm, and Wnt1, Wnt2, Wnt3, Wnt3a, Wnt7a are the main ligands. Inactivation of $\beta$-catenin expression during tooth development leads to disruption of odontoblast differentiation and root formation, whereas its overexpression results in excessive dentin production from mature odontoblasts, indicating that canonical Wnt/ $\beta$-catenin signaling plays important roles in odontoblast development and maturation [26, 27]. We treated mouse neonatal tooth germ mesenchymal cells with CHIR99021, a specific and reversible inhibitor of GSK3 $\beta$ activity. Inhibition of GSK3 $\beta$ by 


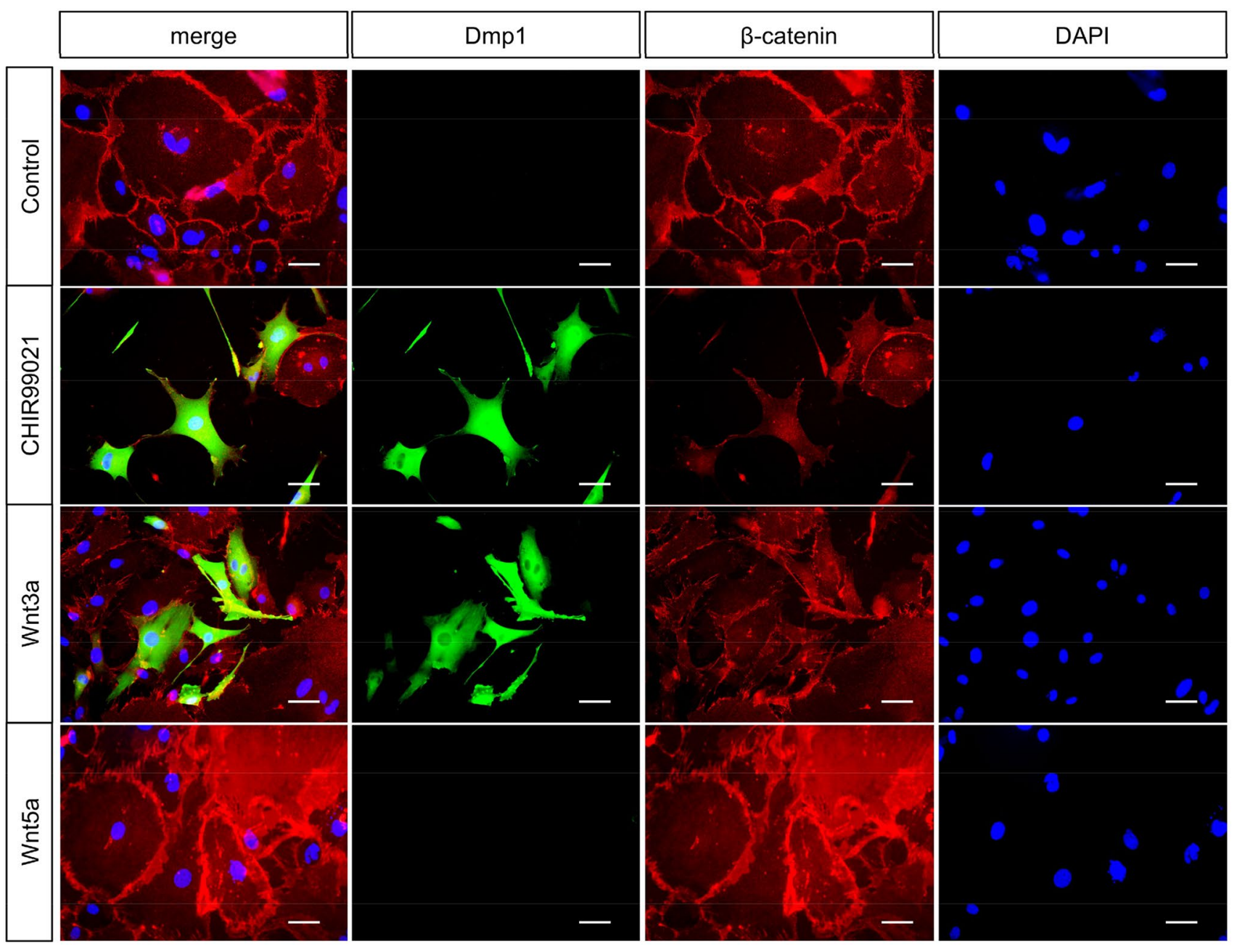

Fig. 3 Promotion of $\beta$-catenin nuclear translocation by administration of CHIR99021. Cells were cultured in medium containing a Wnt signaling activator, and analysis of Dmp1-EGFP and $\beta$-catenin immunofluorescence was performed. Dmp1-EGFP expression was induced

CHIR99021 has been shown to result in activation of the Wnt signaling pathway, as well as sustained pluripotency of human and mouse embryonic stem cells [16]. We found that CHIR99021 treatment induced expression of Dmp1, an odontoblast marker protein. The Cre-LoxP reporter system was utilized to detect Dmp1 expression and that was found in neonatal primary cultured mesenchymal cells from the tooth germ, which were observed to be negative for Dmp1-EGFP expression, as that was not observed until 2 days after birth. Primary cultured tooth germ mesenchymal cells were successfully established and Wnt3a treatment was found to have some effect to induce EGFP expression linked with Dmp1 expression, whereas Wnt5a did not. Expression of Wnt3a is reported in Hertwig's epithelial root sheath of the 2-weekold mice, but not enough research in the early stages of tooth germ formation. These observations suggested that canonical Wnt/ $\beta$-catenin signaling positively supports odontoblast by activation of canonical Wnt signaling. In addition, $\beta$-catenin nuclear translocation was observed with CHIR99021 treatment. Scale bar $=50 \mu \mathrm{m}$

genesis by Wnt3a. In previous studies, we investigated Dspp, another important odontoblast marker [28, 29], and found only a modest increase in expression, indicating that canonical Wnt/ $\beta$-catenin signaling was not enough to induce odontoblast genesis. Other reports have noted that the Wnt/ $\beta$-catenin pathway has a positive effect on Runx2 expression by osteoblasts and drives maturation [30, 31]. Thesleff et al. reported that canonical Wnt signaling induced Runx2 expression in odontoblasts, while Runx2 appeared to inhibit differentiation [32]. Together with those previous reports, the present results indicate that canonical Wnt/ $\beta$-catenin can sustain Runx2 expression expressed by both odontoblasts and osteoblasts, which in turn is speculated to suppress the odontoblast-specific marker protein Dspp. Wnt has at least two intracellular signaling pathways named canonical and non-canonical pathway. Wnt5a is one of the ligands for the non-canonical pathway, while Wnt3a is one of those for 
Fig. 4 Administration of CHIR99021 with or without

FGF8. a Photographs show cells cultured with a single administration of the CHIR99021 or FGF8, as well as both for 3 weeks. Cells with both the CHIR99021 and FGF8 added exhibited a spindle shape. $\mathbf{b}$ Comparison of cell growth curves with single administration of CHIR99021 or FGF8, and both. The values are shown as mean $\pm \mathrm{SD}$ from three independent experiments. $* p<0.01$

a

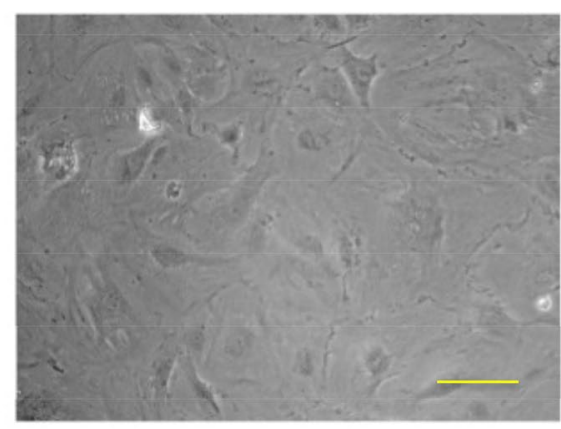

Control

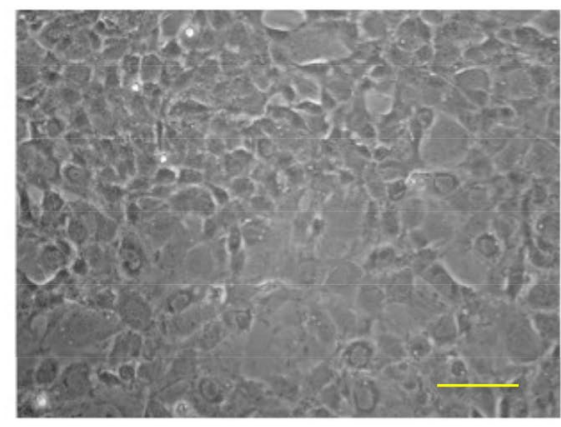

FGF8

b

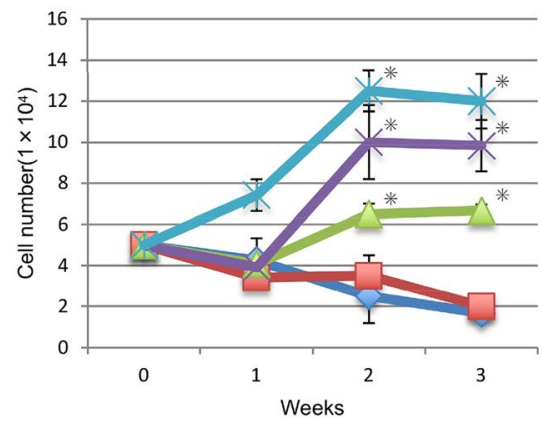

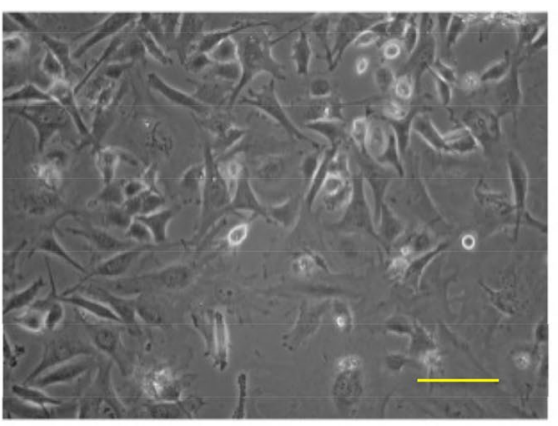

CHIR99021

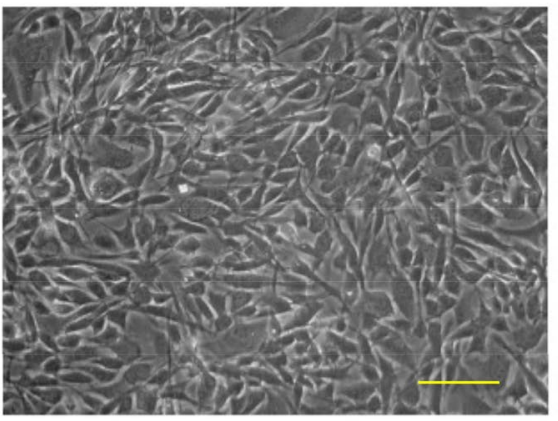

CHIR99021 + FGF8

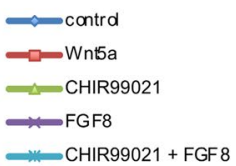

the canonical pathway. Wnt5a regulates cell proliferation, migration, and polarization [33], and the non-canonical Wnt pathway has been shown to inhibit canonical Wnt/ $\beta$-catenin signaling by promoting degradation of $\beta$-catenin in a GSK3independent manner [34]. CHIR99021 is a small molecular compound that inhibits $\beta$-catenin, a downstream component of the canonical Wnt pathway. The present results indicate that when Wnt3a has a tendency to induce odontogenic differentiation. Furthermore, CHIR99021 showed significant upregulation though Wnt5a did not. In addition, it was demonstrated that activation of the canonical Wnt pathway is important for inducing odontogenic differentiation. Therefore, it is speculated that CHIR99021, which is less susceptible to negative feedback than cytokines, induces stronger dental differentiation.

FGF8 in the oral ectoderm defines tooth formation sites by activating expression of the dental mesenchymal marker $\operatorname{Pax} 9$ and has been reported to be a pro-odontogenic factor [11], thus we considered that it may also be an early odontogenic specifier. The present results showed that FGF8 had positive effects on proliferation as well as Dspp expression, while it also induced a reduced level of Runx 2 expression, which likely has positive effects on odontoblastic differentiation. The functions of FGF8 are diverse, including mitogenic/proliferative, as well as regulatory morphological effects. As shown in Fig. 4, FGF8 treatment alone induced a cobblestone-like appearance of tooth germ mesenchymal cells, and those treated with both CHIR99021 and FGF8 showed significantly enhanced growth and a spindle-like morphology. In addition to morphological and proliferative effects, significant increases in osteogenic markers Dmpl, $\beta$-catenin, Alp and $O s x$, were also observed. Also, combined treatment with CHIR99021 and FGF8 resulted in remarkable increases in odontogenic-specific Dmpl, Dspp, Nestin, and Pannexin3. Wnt, FGF, and BMP are known to be interconnected for 

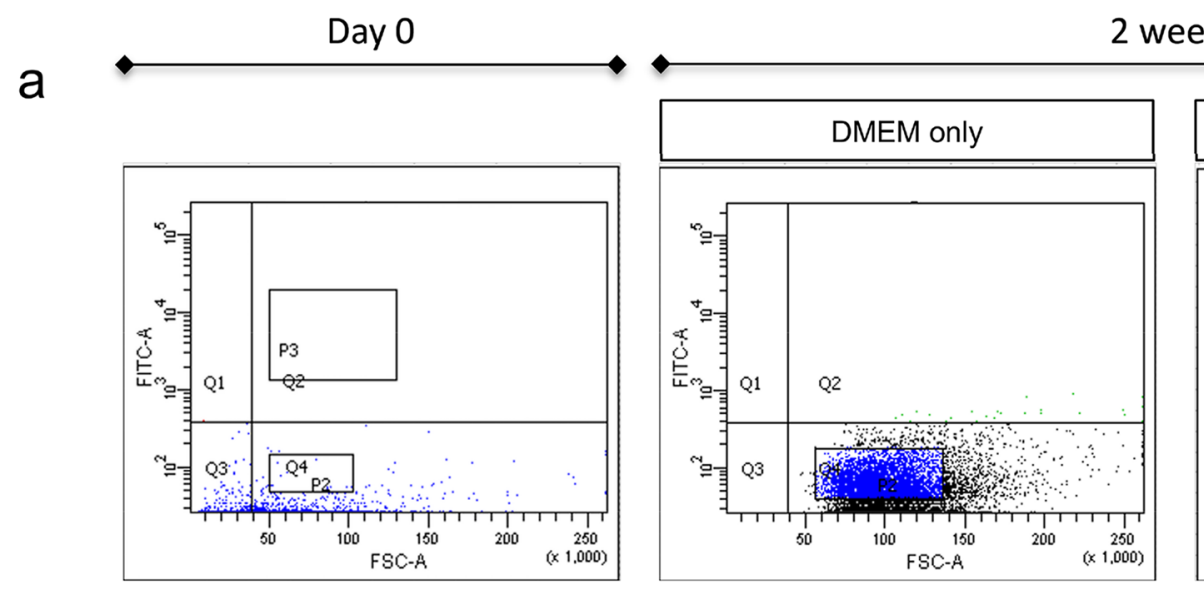

\section{2 weeks}

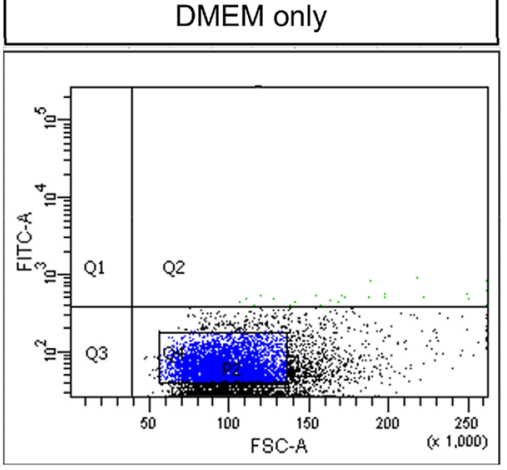

$\mathrm{DMEM}+\mathrm{CHIR99021}+\mathrm{FGF} 8$
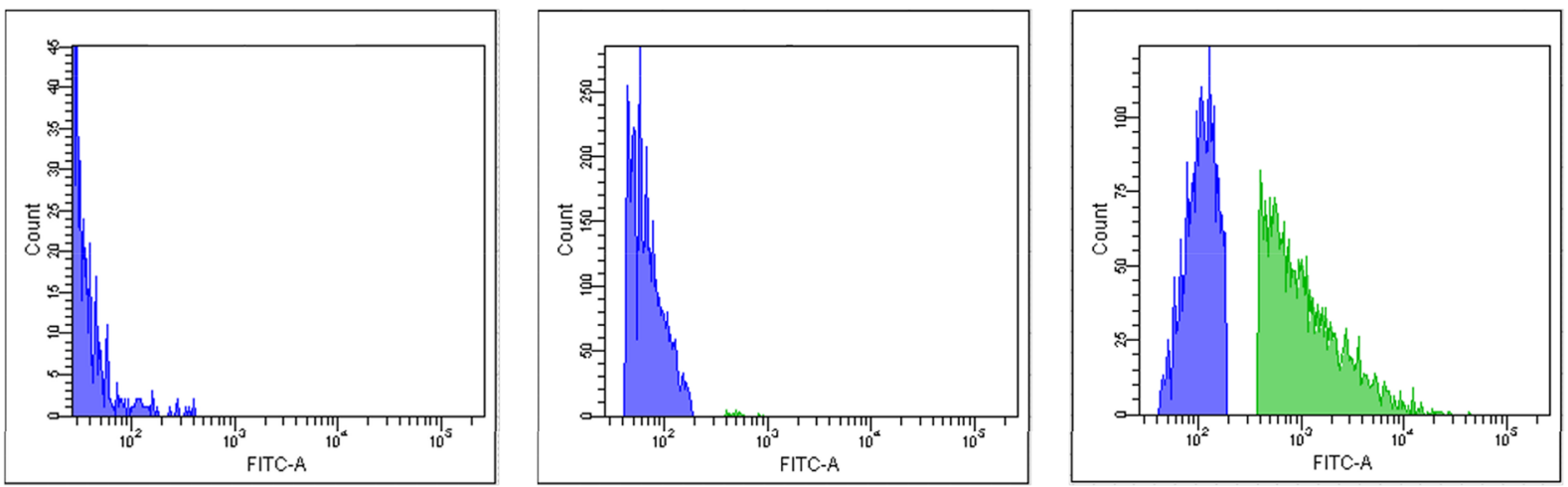

b

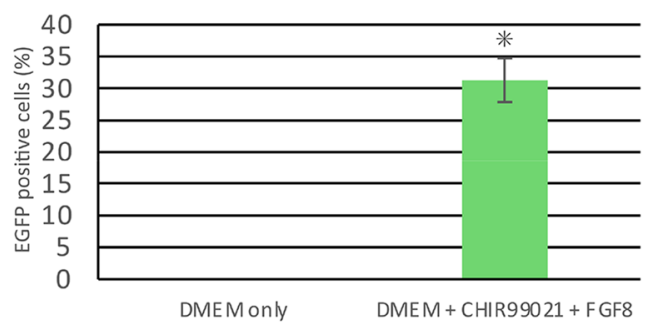

Fig. 5 Isolation of cultured cells using flow cytometry. a Addition of the CHIR99021 with FGF8 induced Dmp1-EGFP expression in cells subjected to flow cytometry analysis. Upper graph: vertical axis indicates EGFP fluorescence intensity and the horizontal axis cell size. Lower graph: vertical axis indicates cell number and the horizontal axis cell size. Blue, Dmp1-EGFP negative cells. Green, Dmp1-EGFP positive cells. b Quantification of Dmp1-EGFP positive cells by flow cytometry. Cells were cultured with or without CHIR99021 and FGF8 as indicated. Blue, Dmp1-EGFP negative cells. Green, Dmp1EGFP positive cells. ${ }^{*} p<0.01$ dental development, and $F G F 9$ and $F G F 10$ are also associated with the Wnt pathway during odontogenesis [35]. However, that study only found co-localization or canonical Wnt pathway enhancement of FGF expression. The present report is the first to show that simultaneous treatment with FGF8 and CHIR99021 significantly enhances odontoblastic differentiation. Our results also indicate that this combination treatment has preferential effects on odontoblast genesis, as that produced different changes in osteoblast markers as well as increased the expression of all odontoblast markers.

\section{Conclusion}

In summary, Dmpl was found to have a well-differentiated expression in odontoblasts. Pre-matured tooth germ mesenchymal cells expressed neither Dmpl nor Dspp. It was suggested that concurrent stimulation of Wnt and FGF8 signaling induces differentiation of dental mesenchymal cells into odontoblast-like cells. Although additional detailed investigations are needed, the present 
a
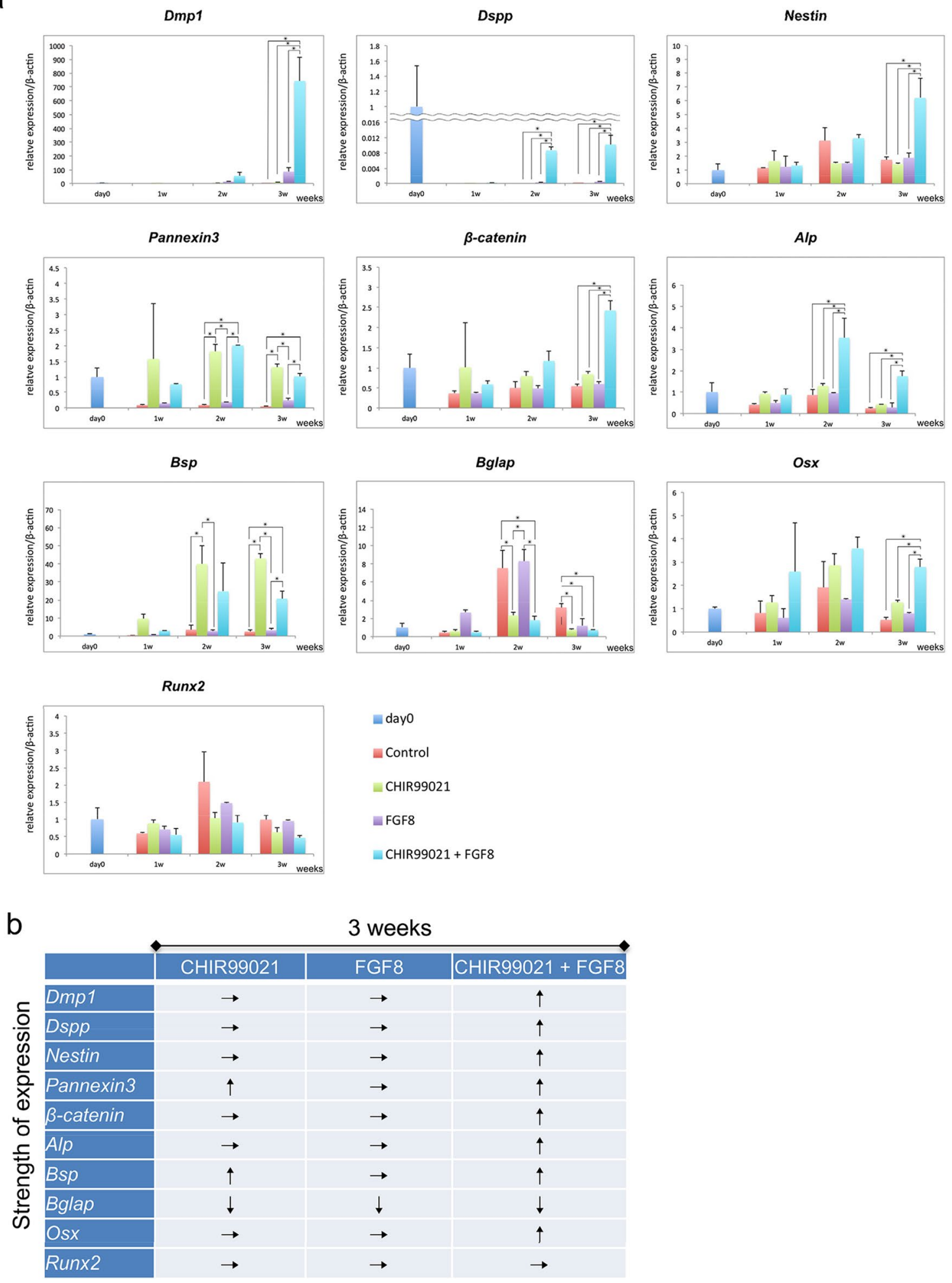

3 weeks

Fig. 6 Evaluation of odontoblast differentiation as effects of CHIR99021 and FGF8. a Shown are mRNA levels of dental osteo/ odonto-related genes in freshly isolated mDMCs (day 0) as well as mDMCs cultured in CHIR99021-, FGF8-, and CHIR99021/FGF8- supplemented medium. ${ }^{*} p<0.01$. b mRNA expression schema of examined genes after 3 weeks. Arrows indicate expression intensity of each sample as compared to control 
observations provide important cues for a more detailed elucidation of odontoblast genesis.

Funding This study was supported by Japan Society for the Promotion of Science: 18H03007 and 19K19074. Genetic engineering of mice was partly supported by JST ERATO Suematsu Gas Biology (2010-2015, M.S. as the lead). The funders had no role in study design, data collection and analysis, decision to publish, or preparation of the manuscript.

\section{Declarations}

Conflict of interest The authors declare that they have no conflict of interest.

Open Access This article is licensed under a Creative Commons Attribution 4.0 International License, which permits use, sharing, adaptation, distribution and reproduction in any medium or format, as long as you give appropriate credit to the original author(s) and the source, provide a link to the Creative Commons licence, and indicate if changes were made. The images or other third party material in this article are included in the article's Creative Commons licence, unless indicated otherwise in a credit line to the material. If material is not included in the article's Creative Commons licence and your intended use is not permitted by statutory regulation or exceeds the permitted use, you will need to obtain permission directly from the copyright holder. To view a copy of this licence, visit http://creativecommons.org/licenses/by/4.0/.

\section{References}

1. Li J, Parada C, Chai Y (2017) Cellular and molecular mechanisms of tooth root development. Development 144:374-384

2. Duverger O, Morasso MI (2008) Role of homeobox genes in the patterning, specification and differentiation of ectodermal appendages in mammals. J Cell Physiol 216:337-346

3. Thesleff I (2003) Epithelial-mesenchymal signalling regulating tooth morphogenesis. J Cell Sci 116:1647-1648

4. Zhang YD, Chen Z, Song YQ, Liu C, Liu C, Chen YP (2005) Making a tooth: growth factors, transcription factors, and stem cells. Cell Res 15:301-316

5. Bei M (2009) Molecular genetics of tooth development. Curr Opin Genet Dev 19:504-510

6. Thesleff I, Tummers M (2008) Tooth organogenesis and regeneration. StemBook. https://doi.org/10.3824/stembook.1.37.1

7. Jarvinen E, Salazar-Ciudad I, Birchmeier W, Taketo MM, Jernvall J, Thesleff I (2006) Continuous tooth generation in mouse is induced by activated epithelial Wnt/beta-catenin signaling. Proc Natl Acad Sci 103:18627-18632

8. Zhu X, Zhao P, Liu Y, Zhang X, Fu J, Yu J, Yu H, Qiu M, Chen Y, Hsu W, Zhang Z (2013) Intra-epithelial requirement of canonical Wnt signaling for tooth morphogenesis. J Biol Chem 288:12080-12089

9. Chen J, Lan Y, Baek J, Gao Y, Jiang R (2009) Wnt/beta-catenin signaling plays an essential role in activation of odontogenic mesenchyme during early tooth development. Dev Biol 334:174-185

10. Zhang H, Wang J, Deng F, Huang E, Yan Z, Wang Z, Deng Y, Zhang Q, Zhang Z, Ye J, Qiao M, Li R, Wang J, Wei Q, Zhou G, Luu HH, Haydon RC, He T, Deng F (2015) Canonical Wnt signaling acts synergistically on BMP9-induced osteo/odontoblastic differentiation of stem cells of dental apical papilla (SCAPs). Biomaterials 39:145-154
11. Liu C, Gu S, Sun C, Ye W, Song Z, Zhang Y, Chen Y (2013) FGF signaling sustains the odontogenic fate of dental mesenchyme by suppressing-catenin signaling. Development 140:4375-4385

12. Trumpp A, Depew MJ, Rubenstein JLR, Bishop JM, Martin GR (1999) Cre-mediated gene inactivation demonstrates that FGF8 is required for cell survival and patterning of the first branchial arch. Genes Dev 13:3136-3148

13. Zhou C, Yang G, Chen M, He L, Xiang L, Ricupero C, Mao JJ, Ling J (2015) Lhx6 and Lhx8: cell fate regulators and beyond. FASEB J 29:4083-4091

14. Kettunen P, Laurikkala J, Itäranta P, Vainio S, Itoh N, Thesleff I (2000) Associations of FGF-3 and FGF-10 with signaling networks regulating tooth morphogenesis. Dev Dyn 219:322-332

15. Kawamoto S, Niwa H, Tashiro F, Sano S, Kondoh G, Takeda J, Tabayashi K, Miyazaki J (2000) A novel reporter mouse strain that expresses enhanced green fluorescent protein upon Cre-mediated recombination. FEBS Lett 470:263-268

16. Ai Z, Shao J, Wu Y, Yu M, Du J, Shi X, Shi X, Zhang Y, Guo Z (2016) CHIR99021 enhances Klf4 expression through $\beta$-catenin signaling and miR-7a regulation in $\mathrm{J} 1$ mouse embryonic stem cells. PLoS ONE 11(3):e0150936. https://doi.org/10.1371/journ al.phone. 0150936

17. Cajánek L, Ribeiro D, Liste I, Parish CL, Bryja V, Arenas E (2009) Wnt/beta-Catenin signaling blockade promotes neuronal induction and dopaminergic differentiation in embryonic stem cells. Stem Cells 27(12):2917-2927. https://doi.org/10.1002/stem. 210

18. Almeida M, Han L, Bellido T, Manolagas SC, Kousteni S (2005) Wnt proteins prevent apoptosis of both uncommitted osteoblast progenitors and differentiated osteoblasts by beta-catenindependent and -independent signaling cascades involving $\mathrm{Src} /$ ERK and phosphatidylinositol 3-kinase/AKT. J Biol Chem 280:41342-41351

19. Hasegawa D, Wada N, Yoshida S, Mitarai H, Arima M, Tomokiyo A, Hamano S, Sugii H, Maeda H (2018) Wnt5a suppresses osteoblastic differentiation of human periodontal ligament stem cell-like cells via Ror2/JNK signaling. J Cell Physiol 233(2):1752-1762

20. Fujimori S, Novak H, Weissenböck M, Jussila M, Gonçalves A, Zeller R, Galloway J, Thesleff I, Hartmann C (2010) Wnt/ $\beta$ catenin signaling in the dental mesenchyme regulates incisor development by regulating Bmp4. Dev Biol 348:97-106

21. Bei M, Maas R (1998) FGFs and BMP4 induce both Msx1-independent and Msx1-dependent signaling pathways in early tooth development. Development 125:4325-4333

22. Arakaki M, Ishikawa M, Nakamura T, Iwamoto T, Yamada A, Fukumoto E, Saito M, Otsu K, Harada H, Yamada Y, Fukumoto S (2012) Role of epithelial-stem cell interactions during dental cell differentiation. J Biol Chem 287:10590-10601

23. Babb R, Chandrasekaran D, Carvalho Moreno Neves V, Sharpe PT (2017) Axin2-expressing cells differentiate into reparative odontoblasts via autocrine $\mathrm{Wnt} / \beta$-catenin signaling in response to tooth damage. Sci Rep 7:3102

24. Mohammed MK, Shao C, Wang J, Wei Q, Wang X, Collier Z, Tang S, Liu H, Zhang F, Huang J, Guo D, Lu M, Liu F, Liu J, Ma C, Shi LL, Athiviraham A, He T, Lee MJ (2016) Wnt/ $\beta$-catenin signaling plays an ever-expanding role in stem cell self-renewal, tumorigenesis and cancer chemoresistance. Genes Dis 3:11-40

25. Dravid G, Ye Z, Hammond H, Chen G, Pyle A, Donovan P, Yu X, Cheng L (2005) Defining the role of Wnt/ $\beta$-catenin signaling in the survival, proliferation, and self-renewal of human embryonic stem cells. Stem Cells 23:1489-1501

26. Aurrekoetxea M, Lopez J, García P, Ibarretxe G, Unda F (2012) Enhanced Wnt/ $\beta$-catenin signalling during tooth morphogenesis impedes cell differentiation and leads to alterations in the structure and mineralisation of the adult tooth. Biol Cell 104:603-617 
27. Zhang R, Yang G, Wu X, Xie J, Yang X, Li T (2013) Disruption of $\mathrm{Wnt} / \beta$-catenin signaling in odontoblasts and cementoblasts arrests tooth root development in postnatal mouse teeth. Int J Biol Sci 9:228-236

28. Zhu Q, Gibson MP, Liu Q, Liu Y, Lu Y, Wang X, Feng JQ, Qin C (2012) Proteolytic processing of dentin sialophosphoprotein (DSPP) is essential to dentinogenesis. J Biol Chem 287:30426-30435

29. Chen Y, Zhang Y, Ramachandran A, George A (2016) DSPP is essential for normal development of the dental-craniofacial complex. J Dent Res 95:302-310

30. Vega OA, Lucero CMJ, Araya HF, Jerez S, Tapia JC, Antonelli M, Salazar-Onfray F, Heras FL, Thaler R, Riester SM, Stein GS, van Wijnen AJ, Galindo MA (2017) Wnt/ $\beta$-catenin signaling activates expression of the bone-related transcription factor RUNX2 in select human osteosarcoma cell types. J Cell Biochem 118:3662-3674

31. Cai T, Sun D, Duan Y, Wen P, Dai C, Yang J, He W (2016) WNT/ $\beta$-catenin signaling promotes VSMCs to osteogenic transdifferentiation and calcification through directly modulating Runx 2 gene expression. Exp Cell Res 345:206-217

32. Lim WH, Liu B, Cheng D, Hunter DJ, Zhong Z, Ramos DM, Williams BO, Sharpe PT, Bardet C, Mah SJ, Helms JA (2014)
Wnt signaling regulates pulp volume and dentin thickness. J Bone Miner Res 29:892-901

33. He F, Xiong W, Yu X, Espinoza-Lewis R, Liu C, Gu S, Nishita M, Suzuki K, Yamada G, Minami Y, Chen Y (2008) Wnt5a regulates directional cell migration and cell proliferation via Ror2-mediated noncanonical pathway in mammalian palate development. Development 135(23):3871-3879

34. Topol L, Jiang X, Choi H, Garrett-Beal L, Carolan PJ, Yang Y (2003) Wnt-5a inhibits the canonical Wnt pathway by promoting GSK-3-independent beta-catenin degradation. J Cell Biol 162(5):899-908

35. Cobourne MT, Sharpe PT (2010) Making up the numbers: the molecular control of mammalian dental formula. Semin Cell Dev Biol 21:314-324

Publisher's Note Springer Nature remains neutral with regard to jurisdictional claims in published maps and institutional affiliations. 